\title{
ON PORTFOLIO OPTIMIZATION UNDER "DRAWDOWN" CONSTRAINTS
}

\author{
JAKSA CVITANIC* IOANNIS KARATZAS
}

March 16, 1994

\begin{abstract}
We study the problem of portfolio optimization under the "drawdown constraint" that the wealth process never falls below a fixed fraction of its maximum-to-date, and one strives to maximize the long-term growth rate of its expected utility. This problem was introduced and solved explicitly by Grossman and Zhou; we present an approach which simplifies and extends their results.
\end{abstract}

\section{Introduction and Summary}

In a very interesting recent article, Grossman \& Zhou (1993) consider the classical portfolio optimization problem of Merton (1971) under the "drawdown constraint" that the wealth process $X^{\pi}(\cdot)$ satisfy:

$$
X^{\pi}(t)>\alpha \max _{0 \leq s \leq t} X^{\pi}(s), \quad \forall 0 \leq t<\infty
$$

almost surely. In other words, one admits only those portfolios $\pi(\cdot)$ for which the corresponding wealth process $X^{\pi}(\cdot)$ never falls below $100 \alpha \%$ of its maximum-to-date, for some given constant

*IMA, University of Minnesota, Minneapolis, MN 55455, on leave from Columbia University, cvitanic@ima.umn.edu

${ }^{\dagger}$ Department of Statistics, Columbia University, New York, NY 10027, ik@stat.columbia.edu 
$\alpha \in(0,1)$. The objective is then to maximize the long-term growth rate

$$
\mathcal{R}(\pi):=\varlimsup_{T \rightarrow \infty} \frac{1}{T} \log E\left(X^{\pi}(T)\right)^{\delta}
$$

of expected utility, for some power $\delta \in(0,1)$, over portfolio rules $\pi(\cdot)$ that satisfy $(1.1)$.

Using a mixture of analytical and probabilistic arguments, Grossman and Zhou provide an explicit solution to this problem, when investment is between a bond and one stock (modeled by geometric Brownian motion with constant coefficients). They show that the optimal portfolio $\hat{\pi}(\cdot)$ always invests a constant proportion of the difference $X^{\hat{\pi}}(t)-\alpha \cdot \max _{0 \leq s \leq t} X^{\hat{\pi}}(t), 0 \leq t<$ $\infty$, in the risky asset. Their insights are impressive, but the arguments are rather lengthy and detailed.

We present in this paper an approach to the above problem, which simplifies the results of Grossman \& Zhou (1993) - and extends them to the case of several stocks with general deterministic coefficients. The model and the problem are introduced in sections 2 and 3 , respectively. The approach is based on an auxiliary finite-horizon stochastic control problem, formulated in section 4 (Problem 4.1, Remark 4.2) in terms of the process $N_{\alpha}^{\pi}(\cdot)$ in (4.1). This problem admits an explicit optimal portfolio $\hat{\pi}(\cdot)$, which is independent of the time-horizon $T \in(0, \infty)$ and can be found using "classical" martingale and duality arguments. It is then a relatively straightforward matter to show that this portfolio $\hat{\pi}(\cdot)$ is also optimal for the problem of maximizing (1.2); this is carried out in section 5. In section 6 we find the optimal portfolio for the case of logarithmic utility function, and in section 7 we show that the same portfolio maximizes the long-term growth rate almost surely, not only in expectation. Moreover, this portfolio is optimal even if we allow random (adapted) market coefficients.

\section{The Model}

Let us consider the following, by now standard, model of a financial market $\mathcal{M}$ with one riskless asset ("bond", price $P_{0}(t)$ at time $t$ ) and a risky assets ("stocks"; prices $P_{i}(t)$ at time $t, 1 \leq i \leq d)$, modeled by the stochastic equations

$$
\begin{aligned}
& d P_{0}(t)=P_{0}(t) r(t) d t, P_{0}(0)=1 \\
& d P_{i}(t)=P_{i}(t)\left[b_{i}(t) d t+\sum_{j=1}^{d} \sigma_{i j}(t) d W_{j}(t)\right], P_{i}(0)=p_{i}>0,
\end{aligned}
$$

for $i=1, \ldots, d$. These equations are driven by the $d$-dimensional Brownian motion $W=$ $\left(W_{i}, \ldots, W_{d}\right)^{\prime}$, which generates all the randomness in the model - in the sense that the interest rate $r(\cdot)$, the vector of appreciation rates $b(\cdot)=\left(b_{1}(\cdot), \ldots, b_{d}(\cdot)\right)^{\prime}$ and the volatility matrix $\sigma(\cdot)=\left\{\sigma_{i j}(\cdot)\right\}_{1 \leq i, j \leq d}$ are bounded measurable processes, adapted to the augmentation $\mathbb{F}=$ 
$\{\mathcal{F}(t)\}_{0 \leq t<\infty}$ of the filtration $\mathcal{F}^{W}(t)=\sigma(W(s), 0 \leq s \leq t), 0 \leq t<\infty$ generated by $W$. We shall assume throughout that the matrix $\sigma(\cdot)$ is invertible, and the "relative risk" process $\theta(t):=\sigma^{-1}(t)[b(t)-r(t) \mathbb{I}], 0 \leq t<\infty$ is bounded as well, where $\mathbb{I}$ is a $d$-dimensional vector with all entries equal to one. We shall also denote by

$$
\beta(t):=\frac{1}{P_{0}(t)}=\exp \left(-\int_{0}^{t} r(s) d s\right), \quad 0 \leq t<\infty
$$

the "discount process" of this model.

Consider now an economic agent who invests in this market according to a portfolio rule $\pi(\cdot)=\left(\pi_{1}(\cdot), \ldots, \pi_{d}(\cdot)\right)^{\prime}$ in such a way that his corresponding wealth process $X^{\pi}(\cdot)$ is governed by the equation

$$
\begin{aligned}
& d X^{\pi}(t)=\sum_{i=1}^{d} \pi_{i}(t)\left(X^{\pi}(t)-\frac{\alpha M^{\pi}(t)}{\beta(t)}\right)\left[b_{i}(t) d t+\sum_{j=1}^{d} \sigma_{i j}(t) d W_{j}(t)\right] \\
& +\left[\left(1-\sum_{i=1}^{d} \pi_{i}(t)\right)\left(X^{\pi}(t)-\frac{\alpha M^{\pi}(t)}{\beta(t)}\right)+\alpha \frac{M^{\pi}(t)}{\beta(t)}\right] r(t) d t \\
& =r(t) X^{\pi}(t) d t+\left(X^{\pi}(t)-\frac{\alpha M^{\pi}(t)}{\beta(t)}\right) \pi^{\prime}(t)[(b(t)-r(t) \mathbb{I}) d t+\sigma(t) d W(t)], \\
& X^{\pi}(0)=x,
\end{aligned}
$$

and satisfies the "drawdown constraint"

$$
P\left[\beta(t) X^{\pi}(t)>\alpha M^{\pi}(t), \quad \forall 0 \leq t<\infty\right]=1 .
$$

Here $\alpha \in(0,1)$ is a given constant, and

$$
M^{\pi}(t):=\max _{0 \leq s \leq t}\left(\beta(s) X^{\pi}(s)\right)
$$

The interpretation is this: the agent does not tolerate the "drawdown $1-\frac{\beta(t) X^{\pi}(t)}{M^{\pi}(t)}$ of his discounted wealth, from its maximum-to-date", to be greater than or equal to the constant $1-\alpha$, at any time $t \geq 0$; thus, he imposes the (almost sure) constraint (2.5). He invests a proportion $\pi_{i}(t)$ of the difference $X^{\pi}(t)-\alpha \frac{M^{\pi}(t)}{\beta(t)}>0$ in the $i$ th stock, $i=1, \ldots, d$, and invests the remainder $\left(1-\sum_{i=1}^{d} \pi_{i}(t)\right)\left(X^{\pi}(t)-\alpha \frac{M^{\pi}(t)}{\beta(t)}\right)+\alpha \frac{M^{\pi}(t)}{\beta(t)}$ of his wealth in the bond.

With this interpretation in mind, we set up the formal model as follows.

2.1 Definition: For a given initial capital $x>0$, let $\mathcal{A}_{\alpha}(x)$ denote the class of measurable, $\mathbb{E}$-adapted processes $\pi:[0, \infty) \times \Omega \rightarrow \mathbb{R}^{d}$ which satisfy

$$
\int_{0}^{T}\left\|\pi^{\prime}(t) \sigma(t)\right\|^{2} d t<\infty, \text { a.s. }
$$


for any given $T \in(0, \infty)$, and for which the stochastic functional/differential equation (2.4), (2.6) has a unique $\mathbb{F}$-adapted solution $X^{\pi}(\cdot)$ that obeys the constraint (2.5).

The elements of $\mathcal{A}_{\alpha}(x)$ will be called "admissible portfolio processes".

The class of Definition 2.1 is non-empty. In fact, it is shown in the Appendix that

$$
\left\{\begin{array}{c}
\text { for any measurable, } \mathbb{E} \text {-adapted process } \rho:[0, T] \times \Omega \rightarrow \mathbb{R}^{d}, \\
\hat{\pi}=\left(\rho^{\prime} \sigma^{-1}\right)^{\prime} \text { is an admissible portfolio in } \mathcal{A}_{\alpha}(x), \\
\text { for any } x>0
\end{array}\right\} .
$$

\section{The Grossman-Zhou Problem}

Let $U:(0, \infty) \rightarrow \mathbb{R}$ be a utility function, i.e., a strictly increasing, strictly concave function of class $C^{1}$ with $U^{\prime}(0+)=\infty, U^{\prime}(\infty)=0$ and $U(0+) \geq-\infty$. The convex dual of this function is given by

$$
\tilde{U}(y):=\max _{x>0}[U(x)-x y]=U(I(y))-y I(y), y>0
$$

where $I(\cdot)$ is the inverse of $U^{\prime}(\cdot)$.

3.1 Problem (Grossman $\&$ Zhou (1993)): For some given $0<\delta<1$, maximize the long-term rate of growth

$$
\mathcal{R}(\pi):=\varlimsup_{T \rightarrow \infty} \frac{1}{T} \log E\left(X^{\pi}(T)\right)^{\delta}
$$

of expected power-utility, over $\pi \in \mathcal{A}_{\alpha}(x)$. In particular, compute

$$
v(\alpha):=\sup _{\pi \in \mathcal{A}_{\alpha}(x)} \mathcal{R}(\pi)
$$

and find $\hat{\pi} \in \mathcal{A}_{\alpha}(x)$, for which the limit $\lim _{T \rightarrow \infty} \frac{1}{T} \log E\left(X^{\hat{\pi}}(T)\right)^{\delta}=\mathcal{R}(\hat{\pi})$ exists and achieves the supremum in $(3.3)$.

Grossman and Zhou solved Problem 3.1 for $d=1$ and constant $r, b_{1}, \sigma_{11}$, using rather lengthy analytical and probabilistic techniques. We present in section 5 a simple solution to this problem that allows general $d \geq 1$ and deterministic coefficients $r(\cdot), b(\cdot), \sigma(\cdot)$ in $(2.1)$, $(2.2)$.

\section{An Auxiliary Process and Problem}

For any portfolio process $\pi \in \mathcal{A}_{\alpha}(x)$ as in Definition (2.1), consider the auxiliary process

$$
N_{\alpha}^{\pi}(t):=\left(X^{\pi}(t)-\alpha \frac{M^{\pi}(t)}{\beta(t)}\right)\left(M^{\pi}(t)\right)^{\frac{\alpha}{1-\alpha}}, \quad 0 \leq t<\infty .
$$


Because the increasing process $M^{\pi}(\cdot)$ of (2.6) is flat off the set $\left\{t \geq 0 / \beta(t) X^{\pi}(t)=M^{\pi}(t)\right\}$, we have from $(2.4),(2.3),(4.1)$ :

$$
d\left(\beta(t) N_{\alpha}^{\pi}(t)\right)=\left(\beta(t) N_{\alpha}^{\pi}(t)\right) \pi^{\prime}(t) \sigma(t) d W_{0}(t), \quad W_{0}(t):=W(t)+\int_{0}^{t} \theta(s) d s .
$$

Consider also the processes

$$
Z(t):=\exp \left\{-\int_{0}^{t} \theta^{\prime}(s) d W(s)-\frac{1}{2} \int_{0}^{t}\|\theta(s)\|^{2} d s\right\}, \quad H(t):=\beta(t) Z(t) .
$$

From the product rule $d\left(H(t) N_{\alpha}^{\pi}(t)\right)=\beta(t) N_{\alpha}^{\pi}(t) d Z(t)+Z(t) d\left(\beta(t) N_{\alpha}^{\pi}(t)\right)+d\left\langle Z, \beta N_{\alpha}^{\pi}\right\rangle(t)$ and (4.2), (4.3) we obtain: $d\left(H(t) N_{\alpha}^{\pi}(t)\right)=H(t) N_{\alpha}^{\pi}(t)\left(\pi^{\prime}(t) \sigma(t)-\theta^{\prime}(t)\right) d W(t)$. In other words, for any $\pi \in \mathcal{A}_{\alpha}(x)$ the process

$$
H(t) N_{\alpha}^{\pi}(t)=(1-\alpha) x^{\frac{1}{1-\alpha}} \exp \left\{\int_{0}^{t}\left(\pi^{\prime} \sigma-\theta^{\prime}\right)(s) d W(s)-\frac{1}{2} \int_{0}^{t}\left\|\pi^{\prime} \sigma-\theta^{\prime}\right\|^{2}(s) d s\right\}
$$

is a positive local martingale, hence supermartingale, which thus satisfies

$$
E\left[H(T) N_{\alpha}^{\pi}(T)\right] \leq(1-\alpha) x^{\frac{1}{1-\alpha}}, \quad \forall T \in(0, \infty) .
$$

We now pose an auxiliary stochastic control problem, involving the process $N_{\alpha}^{\pi}(\cdot)$ of $(4.1)$.

4.1 An Auxiliary, Finite-Horizon, Control Problem: For a given $T \in(0, \infty)$ and utility function $U:(0, \infty) \rightarrow \mathbb{R}$, denote by $\mathcal{A}_{\alpha}(x, T)$ the class of portfolios $\pi(\cdot)$ that satisfy the requirements of Definition 2.1 on the finite horizon $[0, T]$, and find $\hat{\pi}(\cdot) \in \mathcal{A}_{\alpha}(x, T)$ that achieves

$$
V(\alpha ; T, x):=\sup _{\pi \in \mathcal{A}_{\alpha}(x, T)} \operatorname{EU}\left(N_{\alpha}^{\pi}(T)\right) .
$$

There is a fairly straightforward solution to this problem along the lines of Karatzas, Lehoczky \& Shreve (1987), as follows: For any $y>0, \pi \in \mathcal{A}_{\alpha}(x, T)$ we have from (3.1), (4.5):

$$
E U\left(N_{\alpha}^{\pi}(T)\right) \leq E \tilde{U}(y H(T))+y E\left(H(T) N_{\alpha}^{\pi}(T)\right) \leq E \tilde{U}(y H(T))+y(1-\alpha) x^{\frac{1}{1-\alpha}}
$$

The inequalities in (4.7) are equalities, if and only if $y=\hat{y}$ and $\pi(\cdot)=\hat{\pi}(\cdot)$ are such that both

$$
\begin{aligned}
N_{\alpha}^{\hat{\pi}}(T) & =I(\hat{y} H(T)), \\
E[H(T) I(\hat{y} H(T))] & =(1-\alpha) x^{1 / 1-\alpha}
\end{aligned}
$$

hold. Now $\hat{y}$ is uniquely determined from (4.9), and a portfolio $\hat{\pi} \in \mathcal{A}_{\alpha}(x)$ satisfying (4.8) can be found by introducing the positive martingale

$$
\mathcal{Q}(t):=E[H(T) I(\hat{y} H(T)) \mid \mathcal{F}(t)]=(1-\alpha) x^{\frac{1}{1-\alpha}}+\int_{0}^{t} \mathcal{Q}(s) \varphi^{\prime}(s) d W(s), 0 \leq t \leq T .
$$


The second equality follows from the representation theorem for Brownian martingales as stochastic integrals with respect to the Brownian motion $W$ (e.g. Karatzas \& Shreve (1991), $\S 3.4)$, and $\varphi:[0, T] \times \Omega \rightarrow \mathbb{R}^{d}$ is a measurable, $\mathbb{F}$-adapted process with $\int_{0}^{T}\|\varphi(s)\|^{2} d s<\infty$, a.s. Comparing (4.10) with (4.4) and recalling (2.8), we see that

$$
\hat{\pi}(\cdot)=\left(\left(\theta^{\prime}+\varphi^{\prime}\right) \sigma^{-1}\right)^{\prime}(\cdot) \in \mathcal{A}_{\alpha}(x, T), \quad H(\cdot) N^{\hat{\pi}}(\cdot)=\mathcal{Q}(\cdot), a . s .
$$

In particular, (4.8) follows, and

$$
V(\alpha ; T, x)=E[(U \circ I)(\hat{y} H(T))]
$$

4.2 Remark: Let us consider now Problem 4.1 with utility function

$$
U(x)=\frac{1}{\gamma} x^{\gamma} \quad \text { for } \gamma:=\delta(1-\alpha), \quad 0<\delta<1 .
$$

Then, with $\mu:=\frac{\gamma}{1-\gamma}$, the formulae (4.9), (4.12) become

$$
\hat{y}^{-\frac{1}{1-\gamma}}=\frac{(1-\alpha) x^{-\frac{1}{1-\alpha}}}{E\left[(H(T))^{-\mu}\right]}, \quad V(\alpha ; T, x)=\frac{1}{\gamma}\left((1-\alpha) x^{\frac{1}{1-\alpha}}\left(E(H(T))^{-\mu}\right)^{1 / \mu}\right)^{\gamma} .
$$

If, in addition, the coefficients $r(\cdot), b(\cdot), \sigma(\cdot)$ are deterministic, then

$$
(H(t))^{-\mu}=\exp \left[\mu \int_{0}^{t} \theta^{\prime}(s) d W(s)-\frac{\mu^{2}}{2} \int_{0}^{t}\|\theta(s)\|^{2} d s\right] \exp \left\{\mu \int_{0}^{t}\left(r(s)+\frac{1+\mu}{2}\|\theta(s)\|^{2}\right) d s\right\}
$$

and (4.10), (4.11), (4.14) give

$$
\begin{gathered}
\mathcal{Q}(t)=(1-\alpha) x^{\frac{1}{1-\alpha}} \exp \left\{\mu \int_{0}^{t} \theta^{\prime}(s) d W(s)-\frac{\mu^{2}}{2} \int_{0}^{t}\|\theta(s)\|^{2} d s\right\}, \quad \varphi(t)=\mu \theta(t) \\
\hat{\pi}^{\prime}(t) \sigma(t)=(1+\mu) \theta^{\prime}(t)=\frac{1}{1-\delta(1-\alpha)} \theta^{\prime}(t), \quad \text { independent of } T \\
V(\alpha ; T, x)=\frac{1}{\gamma}\left((1-\alpha) x^{\frac{1}{1-\alpha}} \exp \left\{\int_{0}^{T}\left(r(t)+\frac{1+\mu}{2}\|\theta(t)\|^{2}\right) d t\right\}\right)^{\gamma}
\end{gathered}
$$

Clearly, the portfolio $\hat{\pi}(\cdot)$ of $(4.16)$ is well-defined for all $0 \leq t<\infty$; it belongs to $\mathcal{A}_{\alpha}(x)$ of Definition 2.1 for any $x \in(0, \infty)$, by $(2.8)$. 


\section{Solution of the Grossman-Zhou Problem}

We shall assume in this section that

$$
\left\{\begin{array}{c}
\text { the coefficients } r(\cdot), b(\cdot), \sigma(\cdot) \text { in the model of }(2.1),(2.2) \text { are deterministic, } \\
\text { and that } r_{*}:=\lim _{T \rightarrow \infty} \frac{1}{T} \int_{0}^{T} r(s) d s,\left\|\theta_{*}\right\|^{2}:=\lim _{T \rightarrow \infty} \frac{1}{T} \int_{0}^{T}\|\theta(s)\|^{2} d s \\
\text { exist and are finite. }
\end{array}\right\}
$$

5.1 Theorem: Under the assumption (5.1), the portfolio $\hat{\pi}(\cdot)$ of (4.16) is optimal for the Problem 3.1. In fact, in the notation of (3.2), (3.3), (4.17) and (5.1) we have

$$
\lim _{T \rightarrow \infty} \frac{1}{T} \log E\left(X^{\hat{\pi}}(T)\right)^{\delta}=\mathcal{R}(\hat{\pi})=v(\alpha)=V(\alpha)+\alpha \delta r_{*}
$$

where

$$
V(\alpha):=\lim _{T \rightarrow \infty} \frac{1}{T} \log V(\alpha ; T, x)=\gamma r_{*}+\frac{\gamma}{2}(1+\mu)\left\|\theta_{*}\right\|^{2}=\delta(1-\alpha)\left[r_{*}+\frac{\left\|\theta_{*}\right\|^{2}}{2} \frac{\delta(1-\alpha)}{1-\delta(1-\alpha)}\right]
$$

In order to establish this result, it will be helpful to consider the auxiliary problem

$$
\bar{v}(\alpha):=\sup _{\pi \in \mathcal{A}_{\alpha}(x)} \overline{\mathcal{R}}_{\alpha}(\pi), \quad \overline{\mathcal{R}}_{\alpha}(\pi):=\varlimsup_{T \rightarrow \infty} \frac{1}{T} \log E\left(N^{\pi}(T)\right)^{\delta(1-\alpha)} .
$$

From the fact that the portfolio $\hat{\pi}(\cdot)$ of $(4.16)$ does not depend on the horizon $T \in(0, \infty)$, it is clear that

$$
\lim _{T \rightarrow \infty} \frac{1}{T} \log E\left(N_{\alpha}^{\hat{\pi}}(T)\right)^{\delta(1-\alpha)}=\overline{\mathcal{R}}_{\alpha}(\hat{\pi})=\bar{v}(\alpha)=V(\alpha) .
$$

It will also be helpful to note from (4.1) that

$$
\left(N_{\alpha}^{\pi}(t)\right)^{\delta(1-\alpha)}=(\beta(t))^{\alpha \delta}\left(X^{\pi}(t)\right)^{\delta}\left(f_{\alpha}\left(\frac{\alpha M^{\pi}(t)}{\beta(t) X^{\pi}(t)}\right)\right)^{\delta},
$$

where the function $f_{\alpha}(x):=\left(\frac{x}{\alpha}\right)^{\alpha}(1-x)^{1-\alpha}, 0 \leq x \leq 1$ is strictly increasing on $(0, \alpha)$ and strictly decreasing on $(\alpha, 1)$.

Proof of Theorem 5.1: From (5.6) we obtain

$$
E\left(N_{\alpha}^{\pi}(T)\right)^{\delta(1-\alpha)} \leq(\beta(T))^{\alpha \delta}(1-\alpha)^{\delta(1-\alpha)} E\left(X^{\pi}(T)\right)^{\delta},
$$

whence

$$
\overline{\mathcal{R}}_{\alpha}(\pi) \leq \mathcal{R}(\pi)-\alpha \delta r_{*} \leq v(\alpha)-\alpha \delta r_{*}, \quad \forall \pi \in \mathcal{A}_{\alpha}(x)
$$


and therefore $V(\alpha) \leq v(\alpha)-\alpha \delta r_{*}$. In order to establish the reverse inequality, take $\eta \in(0, \alpha)$ close enough to $\alpha$ so that $f_{\eta}(\eta) \geq f_{\eta}(\eta / \alpha)$, and observe from (5.6) that for an arbitrary $\pi \in \mathcal{A}_{\alpha}(x)\left(\subseteq \mathcal{A}_{\eta}(x)\right)$ we have

$E\left(N_{\eta}^{\pi}(T)\right)^{\delta(1-\eta)} \geq(\beta(T))^{\eta \delta}\left(f_{\eta}(\eta / \alpha)\right)^{\delta} E\left(X^{\pi}(T)\right)^{\delta}=(\beta(T))^{\eta \delta}\left(\alpha^{-\eta}(1-\eta / \alpha)^{1-\eta}\right)^{\delta} E\left(X^{\pi}(T)\right)^{\delta}$.

Consequently

$$
V(\eta) \geq \overline{\mathcal{R}}_{\eta}(\pi) \geq \mathcal{R}(\pi)-\eta \delta r_{*}, \quad \forall \pi \in \mathcal{A}_{\alpha}(x),
$$

whence $V(\eta) \geq v(\alpha)-\eta \delta r_{*}$; letting $\eta \uparrow \alpha$ and invoking the continuity of the function $V(\cdot)$ in (5.3), we obtain $V(\alpha) \geq v(\alpha)-\alpha \delta r_{*}$ and thus the third equality of (5.2):

$$
v(\alpha)=V(\alpha)+\alpha \delta r_{*}=\delta r_{*}+\frac{\left\|\theta_{*}\right\|^{2}}{2} \frac{(\delta(1-\alpha))^{2}}{1-\delta(1-\alpha)} .
$$

To obtain the second equality in (5.2), it suffices to observe that (5.8), (5.5), (5.10) imply

$$
v(\alpha) \geq \mathcal{R}(\hat{\pi}) \geq \overline{\mathcal{R}}_{\alpha}(\hat{\pi})+\alpha \delta r_{*}=V(\alpha)+\alpha \delta r_{*}=v(\alpha) .
$$

Finally, the first equality in (5.2), i.e., the existence of the indicated limit, follows from the double inequality

$$
\begin{gathered}
-\frac{\delta(1-\alpha)}{T} \log (1-\alpha)+\frac{\alpha \delta}{T} \int_{0}^{T} r(s) d s+\frac{1}{T} \log E\left(N^{\hat{\pi}}(T)\right)^{\delta(1-\alpha)} \leq \frac{1}{T} \log E\left(X^{\hat{\pi}}(T)\right)^{\delta} \\
\leq-\frac{\delta}{T} \log \left(\alpha^{-\eta}\left(1-\frac{\eta}{\alpha}\right)^{1-\eta}\right)+\frac{\eta \delta}{T} \int_{0}^{T} r(s) d s+\frac{1}{T} \log E\left(N^{\hat{\pi}}(T)\right)^{\delta(1-\eta)}
\end{gathered}
$$

(a consequence of $(5.7),(5.9))$ in conjunction with (5.5), by passing to the limit as $T \rightarrow \infty$ and then letting $\eta \uparrow \alpha$.

5.2 Remark: Formally setting $\alpha=0$ in (4.16), we recover the well-known optimal portfolio $\hat{\pi}^{\prime}(t) \sigma(t)=\frac{\theta^{\prime}(t)}{1-\delta}$ for the investment problem without the constraint (2.5), with utility function $U(x)=\frac{1}{\delta} x^{\delta}$ from wealth and deterministic coefficients.

\section{Maximizing Long-term Rate of Expected Loga- rithmic Utility}

The methods of section 4-5 can also be used to show that the portfolio

$$
\pi^{*}(t)=\left(\theta^{\prime}(t) \sigma^{-1}(t)\right)^{\prime}, \quad 0 \leq t<\infty
$$


is optimal for the problem of maximizing the long-term rate of expected logarithmic utility under the constraint (2.5):

$$
\varlimsup_{T \rightarrow \infty} \frac{1}{T} E\left(\log X^{\pi}(T)\right) \leq \lim _{T \rightarrow \infty} \frac{1}{T} E\left(\log X^{\pi_{*}}(T)\right)=(1-\alpha)\left(\bar{r}+\frac{\|\bar{\theta}\|^{2}}{2}\right)+\alpha \bar{r}, \quad \forall \pi \in \mathcal{A}_{\alpha}(x) .
$$

This problem was also considered by Grossman \& Zhou (1993) in their setting. It turns out that $(6.2)$ holds for general random, $\mathbb{E}$-adapted coefficients $r(\cdot), b(\cdot), \sigma(\cdot)$, for which the conditions of section 2 are satisfied and the limits

$$
\bar{r}:=\lim _{T \rightarrow \infty} \frac{1}{T} \int_{0}^{T} \operatorname{Er}(t) d t, \quad\|\bar{\theta}\|^{2}:=\lim _{T \rightarrow \infty} \frac{1}{T} \int_{0}^{T} E\|\theta(t)\|^{2} d t
$$

exist and are finite.

Indeed, consider $u(\alpha)=\sup _{\pi \in \mathcal{A}_{\alpha}(x)} \mathcal{P}(\pi), \mathcal{P}(\pi):=\varlimsup_{1 \rightarrow \infty} \frac{1}{T} E\left(\log X^{\pi}(T)\right)$ and $\bar{u}(\alpha)=\sup _{\pi \in \mathcal{A}_{\alpha}(x)} \overline{\mathcal{P}}_{\alpha}(\pi)$, $\overline{\mathcal{P}}_{\alpha}(\pi):=\varlimsup_{T \rightarrow \infty} \frac{1-\alpha}{T} E\left(\log N_{\alpha}^{\pi}(T)\right)$ instead of the quantities in (3.1), (3.2) and (5.4), respectively. Solving the Problem 4.1 with $U(x)=(1-\alpha) \log x$ leads to $\mathcal{Q}(\cdot) \equiv(1-\alpha) x^{1 / 1-\alpha}, \varphi(\cdot) \equiv 0$ in (4.10), and thus the optimal portfolio of (4.11) takes the form $\pi^{*}(\cdot)$ in $(6.1)$, independent of the finite horizon $T>0$; furthermore, (4.14) becomes $V(\alpha ; T, x)=\log x+\log (1-\alpha)^{1-\alpha}+(1-$ a) $E \int_{0}^{T}\left(r(s)+\frac{1}{2}\|\theta(s)\|^{2}\right) d s$, whence

$$
\bar{u}(\alpha)=\lim _{T \rightarrow \infty} \frac{V(\alpha ; T, x)}{T}=(1-\alpha)\left(\bar{r}+\frac{\|\bar{\theta}\|^{2}}{2}\right) .
$$

Now one writes (5.6) with $\delta=1$, and uses exactly the same methodology as in section 5 to prove that $\lim _{T \rightarrow \infty} \frac{1}{T} E\left(\log X^{\pi_{*}}(T)\right)=\mathcal{P}\left(\pi^{*}\right)=u(\alpha)=\bar{u}(\alpha)+\alpha \bar{r}$, thus establishing $(6.2)$.

\section{Maximization of Long-term Growth Rate from Investment}

More important than the optimality property $(6.2)$, however, is the fact that the portfolio $\pi^{*}(\cdot)$ of (6.1) maximizes the long-term growth rate from investment

$$
\mathcal{S}(\pi):=\varlimsup_{T \rightarrow \infty} \frac{1}{T} \log X^{\pi}(T) \leq \lim _{T \rightarrow \infty} \frac{1}{T} \log X^{\pi^{*}}(T)=(1-\alpha)\left(r^{*}+\frac{\left\|\theta^{*}\right\|^{2}}{2}\right)+\alpha r^{*}, \text { a.s. }
$$

over all $\pi \in \mathcal{A}_{\alpha}(x)$. Again, this comparison is valid for general random, $\mathbb{E}$-adapted coefficients as in section 2 , under the proviso that the limits

$$
r^{*}:=\lim _{T \rightarrow \infty} \frac{1}{T} \int_{0}^{T} r(t) d t, \quad\left\|\theta^{*}\right\|^{2}:=\lim _{T \rightarrow \infty} \frac{1}{T} \int_{0}^{T}\|\theta(t)\|^{2} d t
$$


exist and are finite, almost surely.

In order to prove (7.1), let us start by noticing that $\Lambda(t):=N_{\alpha}^{\pi}(t) / N_{\alpha}^{\pi^{*}}(t), 0 \leq t<\infty$ satisfies the stochastic equation

$$
d \Lambda(t)=\Lambda(t)\left(\pi^{\prime}(t) \sigma(t)-\theta^{\prime}(t)\right) d W(t), \quad \Lambda(0)=1
$$

from (4.2) and Itô's rule, and is thus a positive supermartingale, for any $\pi \in \mathcal{A}_{\alpha}(x)$. It follows readily from this (e.g. Karatzas (1989), p. 1243) that $\varlimsup_{t \rightarrow \infty} \frac{1}{t} \log \Lambda(t) \leq 0$, or equivalently

$$
\begin{aligned}
\overline{\mathcal{S}}_{\alpha}(\pi):=\varlimsup_{T \rightarrow \infty} \frac{1}{T} \log \left(N_{\alpha}^{\pi}(T)\right)^{1-\alpha} & \leq \lim _{T \rightarrow \infty} \frac{1}{T} \log \left(N^{\pi^{*}}(T)\right)^{1-\alpha}= \\
& =(1-\alpha)\left(r^{*}+\frac{\left\|\theta^{*}\right\|^{2}}{2}\right)=: \bar{s}(\alpha) \text {, a.s. }
\end{aligned}
$$

The existence of this last limit, and its value, follow from (A.6) and (7.2). On the other hand, the inequality (5.6) with $\delta=1$ leads to (as in (5.11))

$$
\begin{gathered}
\frac{1}{T} \log \left(N_{\alpha}^{\pi}(T)\right)^{1-\alpha}+\frac{\alpha}{T} \int_{0}^{T} r(s) d s-\frac{1-\alpha}{T} \log (1-\alpha) \leq \frac{1}{T} \log X^{\pi}(T) \\
\leq \frac{1}{T} \log \left(N_{\eta}^{\pi}(T)\right)^{1-\eta}+\frac{\eta}{T} \int_{0}^{T} r(s) d s-\frac{1}{T} \log \left(\alpha^{-\eta}(1-\eta / \alpha)^{1-\eta}\right)
\end{gathered}
$$

almost surely, for any $\pi \in \mathcal{A}_{\alpha}(x) \subseteq \mathcal{A}_{\eta}(x)$ and any $\eta \in(0, \alpha)$ sufficiently close to $\alpha$. In particular, (7.4) gives

$$
\overline{\mathcal{S}}_{\alpha}(\pi)+\alpha r^{*} \leq s(\alpha):=\underset{\pi \in \mathcal{A}_{\alpha}(x)}{\operatorname{esssup}} \mathcal{S}(\pi) \text {, a.s. }
$$

in the notation of (7.1)-(7.3), whence $\bar{s}(\alpha)+\alpha r^{*} \leq s(\alpha)$; similarly,

$$
\mathcal{S}(\pi)-\eta r^{*} \leq \overline{\mathcal{S}}_{\eta}(\pi) \leq \bar{s}(\eta), \text { whence } s(\alpha)-\eta r^{*} \leq \bar{s}(\eta)
$$

and in the limit as $\eta \uparrow \alpha: s(\alpha)-\alpha r^{*} \leq \bar{s}(\alpha)$, a.s. It develops that $s(\alpha)=\bar{s}(\alpha)+\alpha r^{*}=$ $(1-\alpha)\left(r^{*}+\frac{1}{2}\left\|\theta^{*}\right\|^{2}\right)+\alpha r^{*}$, and it remains to show the existence of the limit and the equality in (7.1). But both of these follow by writing the double inequality (7.4) with $\pi \equiv \pi^{*}$, letting $T \rightarrow \infty$ to obtain in conjunction with $(7.3)$

$$
s(\alpha)=\bar{s}(\alpha)+\alpha r^{*} \leq \varliminf_{T \rightarrow \infty} \frac{1}{T} \log X^{\pi^{*}}(T) \leq \varlimsup_{T \rightarrow \infty} \frac{1}{T} \log X^{\pi^{*}}(T) \leq s(\eta),
$$

and then letting $\eta \uparrow \alpha$ to conclude $\lim _{T \rightarrow \infty} \frac{1}{T} \log X^{\pi^{*}}(T)=s(\alpha)$, almost surely. 


\section{A Appendix}

We devote this section to the proof of the claim (2.8). Clearly, it suffices to show that for any $x \in(0, \infty)$ and $\rho(\cdot)$ as in $(2.8)$, the stochastic equation

$$
d \hat{X}(t)=(\hat{X}(t)-\alpha \hat{M}(t)) \rho^{\prime}(t) d W_{0}(t), \quad \hat{M}(t)=\max _{0 \leq s \leq t} \hat{X}(s) ; \quad \hat{X}(0)=x
$$

admits a unique $\mathbb{F}$-adapted solution that satisfies a.s.

$$
\hat{X}(t)>\alpha \hat{M}(t), \quad \forall 0 \leq t<\infty .
$$

For then $\hat{X}(\cdot) / \beta(\cdot)$ coincides with $X^{\hat{\pi}}(\cdot)$, the wealth process corresponding to the portfolio $\hat{\pi}=\left(\rho^{\prime} \sigma^{-1}\right)^{\prime}$ according to $(2.4)$, and $(2.5)$ is satisfied; and vice-versa.

Suppose that $\hat{X}(\cdot)$ is an $\mathbb{F}$-adapted process that satisfies (A.1), (A.2). Following Grossman \& Zhou (1993), p. 269, observe that

$$
\begin{aligned}
d\left(\frac{\hat{X}(t)}{\hat{M}(t)}-\alpha\right) & =\left(\frac{\hat{X}(t)}{\hat{M}(t)}-\alpha\right) \rho^{\prime}(t) d W_{0}(t)-\frac{d \hat{M}(t)}{\hat{M}(t)} \text {, whence } \\
d\left(\log \left(\frac{\hat{X}(T)}{\hat{M}(t)}-\alpha\right)\right) & =\xi(t)-\frac{1}{1-\alpha} \frac{d \hat{M}(t)}{\hat{M}(t)}, \quad \xi(t):=\int_{0}^{t} \rho^{\prime}(s) d W_{0}(s)-\frac{1}{2} \int_{0}^{t}\|\rho(s)\|^{2} d s .
\end{aligned}
$$

Therefore,

$$
0 \leq R(t):=\log (1-\alpha)-\log \left(\frac{\hat{X}(t)}{\hat{M}(t)}-\alpha\right)=-\xi(t)+\log \left(\frac{\hat{M}(t)}{x}\right)^{\frac{1}{1-\alpha}} .
$$

Clearly, the continuous increasing process $K(t):=\log \left(\frac{\hat{M}(t)}{x}\right)^{\frac{1}{1-\alpha}}$ is flat away from the set $\{t \geq 0 / \hat{X}(t)=\hat{M}(t)\}$, i.e., away from the zero-set of the continuous nonnegative process $R(\cdot)$ of (A.3). From the theory of the Skorohod equation (e.g. Karatzas \& Shreve (1991), §3.6) we have then $K(t)=\max _{0 \leq s \leq t} \xi(s)$, and from this and (A.3):

$$
\begin{gathered}
\hat{M}(t) \equiv \tilde{M}(t):=x \exp \left\{(1-\alpha) \max _{0 \leq s \leq t} \xi(s)\right\}, \\
\hat{X}(t) \equiv \tilde{X}(t):=x \exp \left\{(1-\alpha) \max _{0 \leq s \leq t} \xi(s)\right\}\left[\alpha+(1-\alpha) \exp \left\{\xi(t)-\max _{0 \leq s \leq t} \xi(s)\right\}\right] .
\end{gathered}
$$

Notice also (from (A.4), (A.5) and (4.1)) that

$$
\beta(t) N_{\alpha}^{\hat{\pi}}(t)=(\hat{X}(t)-\alpha \hat{M}(t))(\hat{M}(t))^{\frac{\alpha}{1-\alpha}}=(1-\alpha) x^{\frac{1}{1-\alpha}} e^{\xi(t)} .
$$

It is straightforward to check that $\tilde{X}(\cdot)$ satisfies (A.1), (A.2). 


\section{References}

[1] S.J. Grossman and Z. Zhou, Optimal investment strategies for controlling drawdowns, Math. Finance 3 (3), (1993), pp. 241-276.

[2] I. Karatzas, Optimization problems in the theory of continuous trading, SIAM J. Control \& Optimization 27, (1989) pp. 1221-1259.

[3] I. Karatzas, J.P. Lehoczky and S.E. Shreve, Optimal portfolio and consumption decisions for a "small investor" on a finite horizon, SIAM J. Control \& Optimization 25, (1987), pp. 1157-1586.

[4] I. Karatzas and S.E. Shreve, Brownian Motion and Stochastic Calculus (2 $2^{\text {nd }}$ edition), Springer-Verlag, New York (1991).

[5] R.C. Merton, Optimum consumption and portfolio rules in a continuous time model, J. Econom. Theory 3, (1971), pp. 373-413, Erratum: Ibid 6 (1973), pp. 213-214. 